SUBJECT AREAS:

CELL LINEAGE

DIFFERENTIATION

EMBRYONIC INDUCTION

BODY PATTERNING

Received

13 May 2013

Accepted

5 July 2013

Published

24 July 2013

\section{Correspondence and} requests for materials should be addressed to M.K. (mikael.kubista@ img.cas.cz) \title{
Xenopus laevis embryos of 8 to 32-cells reveals developmental asymmetry
}

\author{
Monika Flachsova ${ }^{1,2}$, Radek Sindelka' \& Mikael Kubista ${ }^{1,3}$
}

\begin{abstract}
'Laboratory of Gene Expression, Institute of Biotechnology, Academy of Sciences of the Czech Republic, Prague, Czech Republic, ${ }^{2}$ Charles University in Prague, Faculty of Science, Department of Cell Biology, Prague, Czech Republic, ${ }^{3}$ TATAA Biocenter AB, Göteborg, Sweden.
\end{abstract}

We have measured the expression of 41 maternal mRNAs in individual blastomeres collected from the 8 to 32-cell Xenopus laevis embryos to determine when and how asymmetry in the body plan is introduced. We demonstrate that the asymmetry along the animal-vegetal axis in the oocyte is transferred to the daughter cells during early cell divisions. All studied mRNAs are distributed evenly among the set of animal as well as vegetal blastomeres. We find no asymmetry in mRNA levels that might be ascribed to the dorso-ventral specification or the left-right axis formation. We hypothesize that while the animal-vegetal asymmetry is a consequence of mRNA gradients, the dorso-ventral and left-right axes specifications are induced by asymmetric distribution of other biomolecules, probably proteins.

U nderstanding the formation of the embryonic body plan is essential in developmental biology. It is generally accepted that mechanisms of cellular differentiation driving development are based on differential transcription and translation of key regulatory elements. Recent development of highly sensitive and reliable molecular diagnostic methods allows for precise measurements of genes' transcriptional activities in very small samples including single cells ${ }^{1}$. In an excellent study by Guo et al., mouse embryos from 8 to 64 cells were dissociated into individual blastomeres and gene expression profiles were measured using the high-throughput microfluidic quantitative real-time PCR (qPCR) system BioMark ${ }^{2}$. They found early mouse embryos are composed of three types of cells representing the trophoectoderm, epiblast, and primitive endoderm. The three cell types were readily distinguished by the expression profile of 48 genes. Pluripotency of mouse single blastomeres from preimplantation embryos (1 to 16-cell stages) was also demonstrated with multiplex RT-PCR expression analysis by May et al. ${ }^{3}$. Expression profiles of trophoectoderm markers, inner cell mass markers and stemness markers have also been measured on human single blastomeres from 5 to 8 -cell embryos by Galan et al., using microarray ${ }^{4}$ and in single putative stem cells from human adult ovarian surface epithelium ${ }^{5}$.

The Xenopus oocyte has two differentially colored hemispheres known as animal and vegetal. The separation into the hemispheres creates the first developmental axis of the embryo referred to as animal-vegetal. The darker color of the animal hemisphere is due to accumulation of pigmented granules called melanosomes. The vegetal hemisphere stores yolk and has light color ${ }^{6}$. De novo transcription in the developing Xenopus embryo is silenced until the mid-blastula transition stage (MBT), which takes place after twelve cell divisions post fertilization. All mRNA molecules required for the development into MBT must be present in the oocyte. These were transcribed during oogenesis from solely maternal chromosomes. The maternal mRNAs are asymmetrically distributed along the animal-vegetal axis of the oocyte and direct the specialization of the animal and vegetal parts ${ }^{7-9}$. The second embryonic body axis is formed after fertilization and separates the embryo into a dorsal and a ventral part. In Drosophila embryos the mechanism determining the dorsal-ventral axis is triggered by a concentration gradient of gurken maternal mRNA, which is accumulated at the future dorsal site. Absence of gurken mRNA at the ventral site leads to translocation of transcription factor Dorsal from the cytoplasm into the nuclei, where the Dorsal activates genes that specify the ventral part ${ }^{10}$. The mechanistic details of the dorso-ventral axis formation in zebrafish are not known, but differences between the dorsal and ventral parts appear during the gastrula stage, when the ventral side of the embryo gets thinner than the dorsal side ${ }^{11}$. The formation of the dorso-ventral axis in Xenopus laevis embryo can be discerned already at the 4-cell stage ${ }^{12}$. The sperm enters the oocyte through the animal hemisphere and in about 25 minutes the cortical cytoplasm of the oocyte rotates some 30 degrees relative to the inner cytoplasmic mass in opposite direction to the sperm's entrance ${ }^{13,14}$. Dorsal maternal factors, such as 
the Dishevelled protein ( $\mathrm{Dvl})$, move to the future dorsal side in the vegetal hemisphere of the oocyte ${ }^{15}$. Dvl inactivates gsk $3 \beta$ protein and protects $\beta$-catenin from degradation. The accumulation of $\beta$-catenin in the dorsal site of the embryo promotes local Wnt signaling, which regulates transcription of zygotic $x n r 3$ and siamois, leading to dorsalization of the embryo ${ }^{16,17}$. The first cell division cuts the oocyte through the point of sperm entrance in the animal pole and the groove elongates to the vegetal pole. The second cell division is perpendicular to the first and separates the embryo into the future dorsal and ventral halves. All four blastomeres contain cytoplasm from both the animal and vegetal hemispheres, but vary in size and pigmentation $^{12}$. The third and last important body axis of Xenopus laevis embryos creates the right-left asymmetry, which manifests at the gastrula stage when the production of embryonic mRNAs has been initiated. Maternal vg1 protein is more active in the left side of the embryo and activates the expression of $x n r 1$ (Xenopus nodalrelated 1). Xnr1 induces pitx2 production, which is required for the left side formation. Injection of $\mathrm{vg} 1$ protein to the future right side of the embryo causes left-right deformation manifested by random localization of the heart and of the digestive tube ${ }^{18}$.

We have previously used quantitative real-time PCR (qPCR) expression profiling of cryostat sections ( $\mathrm{qPCR}$ tomography) of a single oocyte to identify transcripts that form gradients along the animal-vegetal axis ${ }^{8,9}$. These transcripts will become differentially distributed among the animal and the vegetal blastomeres formed at the third cell division. The purpose of the present study is to elucidate if there are expression gradients throughout the embryo that also reflect the dorsal-ventral axis specification and the left-right axis formation by measuring expression of selected 41 maternal genes in individual blastomeres collected from Xenopus embryos between 8 to 32 -cell stages.

\section{Results}

First we extensively optimized our experimental procedure for high extraction and reverse transcription yields, and high qPCR efficiencies. We standardized the protocol for minimum technical variation for the expression profiling of single blastomeres. The vegetal blastomeres store yolk, which is strong inhibitor of biochemical reactions and can induce high variation in the reverse transcription yield and compromise qPCR efficiencies. An RNA spike (in vitro transcribed artificial RNA with $3^{\prime}$ polyA tail and 5' cap, TATAA Biocenter) was used to validate the reverse transcription reaction and the qPCR. The spike was added to the RNA extracted from the samples together with the random sequence hexamers and oligo-dT primers for reverse transcription. The amount of reverse transcribed spike was then measured along with the endogenous transcripts. For the optimized protocol the standard deviation of the RNA spike Cq values across all samples was below 0.20 cycles, which evidences excellent reproducibility between sample preparations. Embryos from the same mother also showed high concordance. Our protocol for expression profiling is based on collecting the individual blastomeres in random order and we can only keep track from which hemisphere (animal or vegetal) the different blastomeres originated. This was necessary to avoid introducing bias during collection. With this protocol any dorsoventral (or possibly left-right) asymmetry would be reflected by heterogeneity in expression profiles among the blastomeres collected from the same hemisphere. As markers to probe the formation of the three developmental axes we chose ten maternal transcripts that have been implicated in the dorso-ventral patterning: $d v l 2, d v l 3, \operatorname{lr} p 6$, wnt11, tcf3, gsk3b, ctnnb1(= $\beta$-catenin), foxh1, trim36 and axin $1^{16,19-21}$, one important marker for left-right specification: $v g 1^{18}$, and nine genes implicated in animal-vegetal orientation: $d a z l, c d x 1$ (=xcad2), wnt11, vg1, vegt, trim36, ddx25 (=deadsouth), otx1, and maml1 ${ }^{9,19,22,23}$. We also included 25 mRNAs that have previously been observed in the mature oocyte: $f z d 7, \operatorname{bmp} 2$, pias 1, foxr1, frat1, mapk8, odc1, $18 S$ rRNA, 5S rRNA, cycl, acta, tubb, gapdh, eeflal, RNA polymerase II, U3 snoRNA, par1, oct60, est1, apc, zpc, mos, stat3, and pax $6^{8,9}$.

Expression data were collected for a total of 224 single blastomeres. The data were analyzed with principal component analysis (PCA) and hierarchical clustering, which are the most powerful multivariate methods to classify samples based on the collective expression of multiple genes ${ }^{24}$. PCA clearly separated cells originating from the animal and from the vegetal hemispheres into clusters (Fig. 1), and indicated that the vegetal blastomeres are more heterogeneous than the animal blastomeres. Neither of the clusters, however, divided into subgroups that would indicate additional asymmetry that could be ascribed to the formation of the other body axes (e.g. dorsal-ventral and left-right asymmetry). The PCA results were confirmed by classifications using SOM (not shown) and hierarchical clustering. The hierarchical clustering is presented in a

\section{Principal component analysis}

a

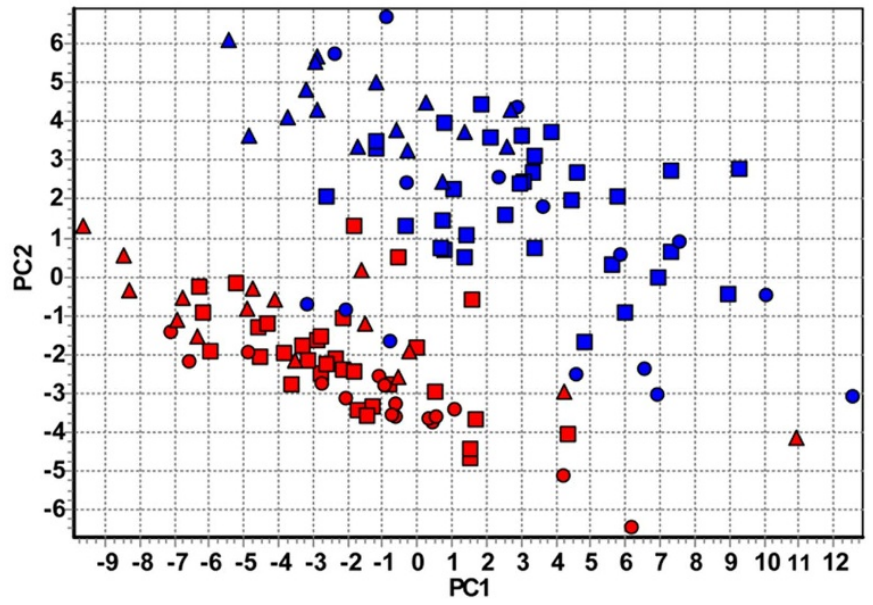

b

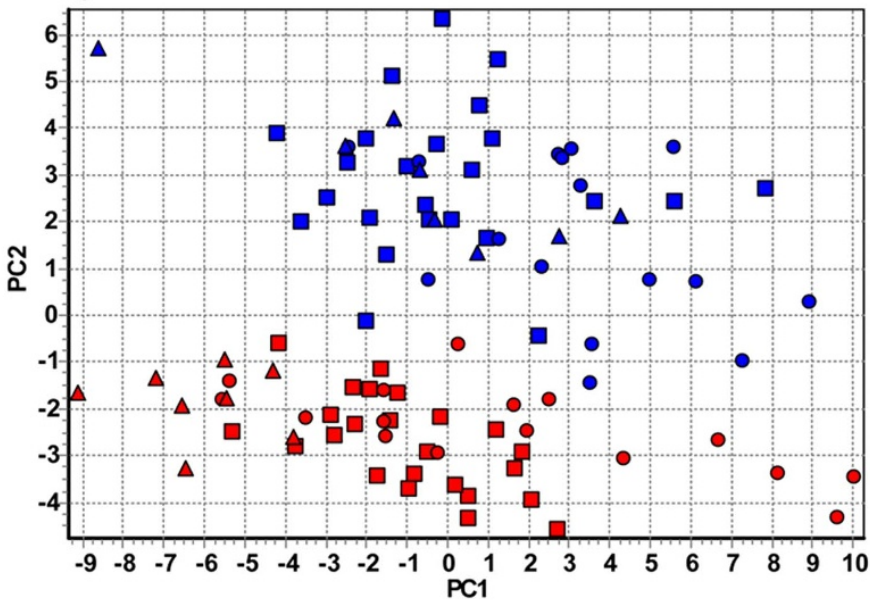

Figure 1 PCA of profiled blastomeres collected from early developmental stages of Xenopus laevis. Triangles indicate blastomeres from the 8-cell

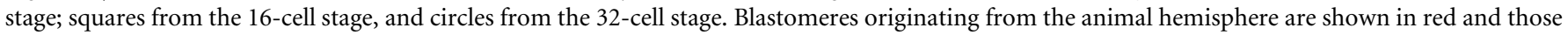
from the vegetal hemisphere are shown in blue. Left and right graphs are data from two different females (left: four embryos from 8-cell stage, four

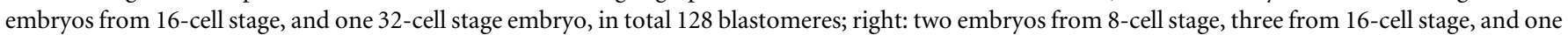
from 32-cell stage, in total 96 blastomeres). 
heatmap (Fig. 2), which is a graphical illustration of all the genes' expressions in all the blastomeres with the level indicated by color, and dendrograms clustering the blastomeres and the genes, respectively, shown on the top and left side. Separate heatmaps were calculated for each embryo from 8-cell (six embryos), 16-cell (seven embryos), and 32-cell (two embryos) stages. Blastomeres of 8 and 16-cell stages separated into well-defined animal and vegetal clusters, but no subclusters that could be ascribed to the formation of dorsalventral or left-right body axes can be discerned. Blastomeres from the 32-cell stage also divided into an animal and a vegetal cluster. The 32cell stage vegetal cluster was diffuse, reflecting heterogeneity among the vegetal blastomeres possibly caused by the blastomeres being arranged into two ventral layers and some transcripts, such as germ plasm determinants, being concentrated in the extreme ventral cortical layer. The genes cluster into four well separated groups at all the developmental stages studied. The first group contains ubiquitously expressed genes represented by $18 S$ rRNA, $5 S$ rRNA, and $c y c 1$. These genes are expressed at high level in all the blastomeres at all stages. A second group is composed of $v g 1, c d x 1$, vegt, dazl, wnt11, otx 1 , trim36, and $d d x 25$, which are most abundant in the vegetal blastomeres. Several of these transcripts have previously been found in the vegetal part of the mature oocyte ${ }^{9}$. Third and fourth groups of transcripts are more abundant in the animal blastomeres, but subtle difference in expression patterns separate them into two clusters. Fzd7, bmp2, pias1, dvl2, dvl3, lrp6, frat1, mapk8, axin1, est1, and U3 snoRNA are expressed at slightly lower level (indicated by the green shade in the heatmap in Fig. 2). Than the fourth set of genes; ctnnb1, foxh1, odc1, zpc, mos, maml1, eef1a1, RNA polymerase II, foxr1, tcf3, gsk3b, oct60, stat3, par1, acta, tubb, gapdh, apc, and pax6 (indicated by the green shade turning to light red in the heatmap in Fig. 2). The separation of genes into these two groups shows some variability across embryos (data not shown). The separation of the gene transcripts into clusters is even more evident in the principal component analysis (PCA) (Fig. 3). One cluster characterizing genes expressed in animal blastomeres: $f z d 7, b m p 2$, pias 1, $d v l 2$, $d v l 3$, $\operatorname{lrp} 6$, frat1, mapk8, axin1, est1, U3 snoRNA, ctnnb1, foxh1, odc1, zpc, mos, maml1, eef1a1, RNA polymerase II, foxr 1, tcf 3, gsk $3 b$, oct 60 , stat3, parl, acta, tubb, gapdh, apc, and pax6; a second cluster of genes expressed in vegatal blastomeres: $v g 1, c d x 1$, vegt, dazl, wnt11, otx1, trim36, and $d d x 25$; a third cluster of high expressed genes: $18 S \mathrm{~S} R N A$, $5 S$ rRNA, and $c y c 1$.

\section{Discussion}

Maternal mRNAs are synthesized during oogenesis and translated during the early development of Xenopus laevis. It is generally accepted that the temporal regulation and the spatial distribution of these maternal mRNAs are important for the establishment of the body axes and subsequently the whole body pattern. The animal-vegetal asymmetry is the first body axis formed during oogenesis. The germ layers, endoderm, mesoderm, and ectoderm, are formed along the animal-vegetal axis such that the vegetal part of the oocyte becomes the endoderm and the most distal animal part of the oocyte gives rise to the ectoderm ${ }^{25,26}$. Transcripts of genes coding specific endodermal and mesodermal factors, such as $v g 1$, wnt 11 , and vegt, are localized in the vegetal hemisphere ${ }^{8,9,22}$. Genes responsible for primordial germ cell formation, such as dazl and $c d x 1$, are localized towards the extreme vegetal pole ${ }^{9}$. Previously we described the new method qPCR tomography to quantify maternal mRNA distribution along the animal-vegetal axis formed during oogenesis, and found two dominant profiles: one with animal and one with vegetal mRNA localization ${ }^{8}$. Subsequent high-resolution qPCR tomography allowed us to discern two vegetal profiles; one encompassing germ plasm determinants ( $d a z l, c d x 1, d d x 25)$ and one encompassing the other vegetal genes investigated $(v g 1 \text {, vegt, wnt } 11 \text {, otx } 1, \text { eg6 })^{9}$. In this study we show that the animal-vegetal polarizations observed in the oocyte remain in the 8,16 , and 32 -cell stage embryos. The vegetal blastomeres had higher levels of $d a z l, c d x 1, d d x 25, v g 1$, vegt, wnt11, ot 1 , as well as trim36, which was not included in the oocyte study. At the 8 and 16-cell stages the vegetal mRNAs were distributed evenly among the vegetal blastomeres; the determinants of the germ plasm being present in all the vegetal cells. At the 32-cell stage, when the vegetal blastomeres form two layers, heterogeneity among the vegetal blastomers was introduced. $18 S$ rRNA, $5 S$ rRNA, cyc1, acta, tubb, gapdh, eef1a1, RNA polymerase II, U3 snoRNA, oct60, axin1, est1, $a p c, t c f 3, z p c, g s k 3 b$, maml1, ctnnb1, mos, foxh1, stat3, fzd7, par7, bmp2, pias1, dvl2, dvl3, lrp6, foxr1, frat1, mapk8, odc1 and pax6 transcripts were more abundant in the animal blastomeres, and were evenly distributed among the individual cells. Multivariate statistical analysis revealed higher variability among the vegetal blastomeres than among the animal. The difference was particularly pronounced at the 32-cell stage. The heterogeneity seems random, possibly caused by yolk platelets that may interfere with PCR inducing technical noise or introduced by the formation of two ventral layers; but we find no evidence for systematic distribution of transcripts neither along the dorsal-ventral axis nor the left-right axis.

An essential question in developmental biology is how amphibians establish the dorsal-ventral axis. The site of sperm entry determines the future ventral half of the embryo. The dorsal part forms on the opposite side and is determined by the accumulation of maternal dorsal determinants during the cortical rotation ${ }^{27-29}$. Consequently, blastomeres of the early Xenopus embryos can be separated into a dorsal and a ventral group with equal number of animal and vegetal cells in each. In Drosophila the dorsalizing determinants have been shown to be maternal mRNA molecules that are asymmetrically distributed within the oocyte and regulate translation that leads to the polarization of the embryo ${ }^{30,31}$. In our study we tested if the dorsalizing factors in Xenopus embryogenesis are also mRNAs coding for dorsal specific proteins within the Wnt pathway. We found that among the maternal transcripts that have been implicated in the induction of dorso-ventral asymmetry ctnnb1, $d v l 2, d v l 3, \operatorname{lrp} 6, g s k 3 b$, tcf3, foxh1, and axin 1, are present predominantly in the animal blastomeres, while trim 36 and $x w n t 11$ are more abundant in the vegetal blastomeres. This supports the idea that the majority of the dorsalventral inducing molecules is localized in the dorsal animal blastomeres of the 16 -cell stage embryo ${ }^{32}$, although we found no evidence of asymmetric distribution of maternal mRNAs among the dorsal or ventral blastomeres. In contrast to Tao et al. ${ }^{33}$, we found wnt11 distributed evenly between the dorsal and ventral cells. Our results rather support the finding of Schroeder et al. ${ }^{34}$, who found maternal wnt11 mRNA symmetrically distributed along the dorsal-ventral axis, and proposed that spatially regulated translation leads to asymmetric distribution of the Wnt11 protein. None of the 41 maternal mRNAs included in this study shows dorso-ventral polarization, although dorso-ventral asymmetry is observed starting from the 4cell stage ${ }^{12}$. Of course, the dorso-ventral asymmetry could be induced by entirely different genes, but since our panel includes ten genes (dvl2, dvl3, lrp6, wnt11, tcf3, gsk3b, ctnnb1, foxh1, trim36, and axin1) previously implicated in dorsal-ventral patterning ${ }^{16,19-21}$, this would be surprising. Rather, we suggest the dorsal-ventral asymmetry in Xenopus laevis is due to early accumulation of dorsal proteins that regulate signaling pathways, which after the midblastula transition activate the expression of downstream dorsalizing factors at the dorsal site of the embryo. We speculate these proteins are present already in the oocyte and accumulate in the future dorsal site during the cortical rotation. Our set includes only one, gene (vg1) previously shown to be involved in the left-right specification ${ }^{18}$. We find no evidence of asymmetric distribution of vgl or of any other of the studied transcripts that could be implicated in left-right specification.

\section{Methods}

Ethics statement. This study was carried out in accordance with the Act No 246/1992 Coll., on the protection of animals against cruelty. Official permission was issued to Faculty of Science, Charles University in Prague by the Central Commission for 


\section{Heatmaps}

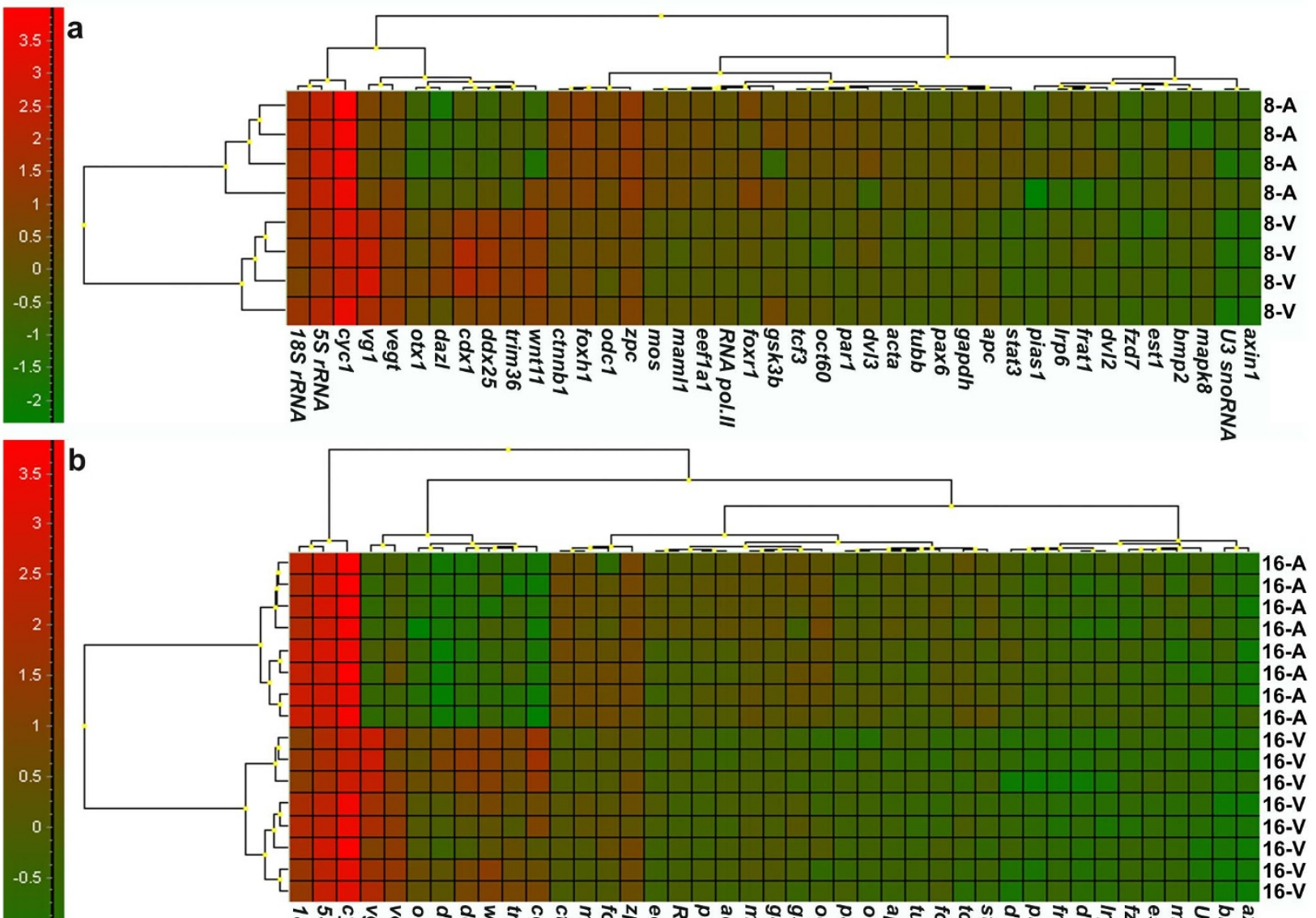

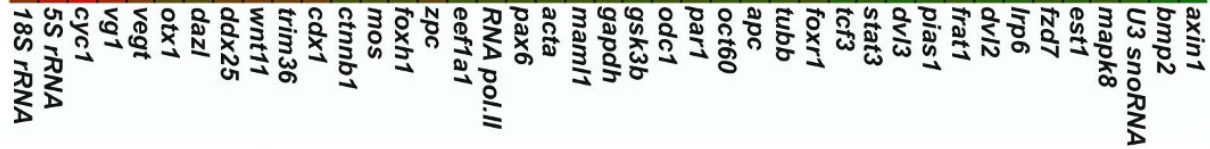
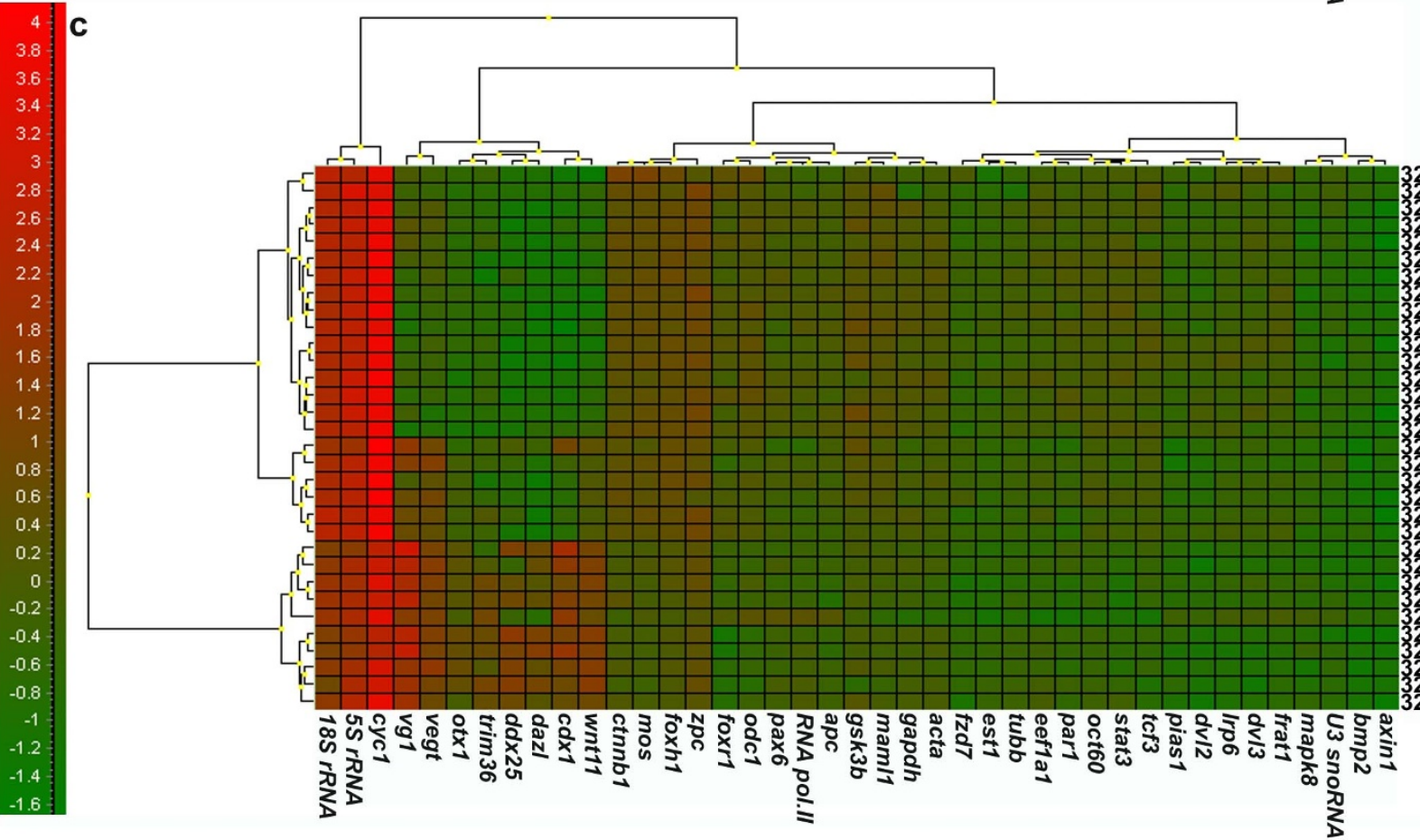

Figure $2 \mid$ Hierarchical clustering of blastomeres and mRNAs of Xenopus laevis early embryos presented as heatmaps. One embryo from each developmental stage was arbitrarily chosen for the cluster analysis. (A) 8-cell, (B) 16-cell and (C) 32-cell embryo. Green color indicates low expression and red high expression. The dendrograms clustering blastomeres and genes are shown in the top and left side of the heatmap, respectively. In the dendrograms similarity between blastomeres/genes is indicated by the height at which they are joined. 


\section{Principal component analysis of genes}
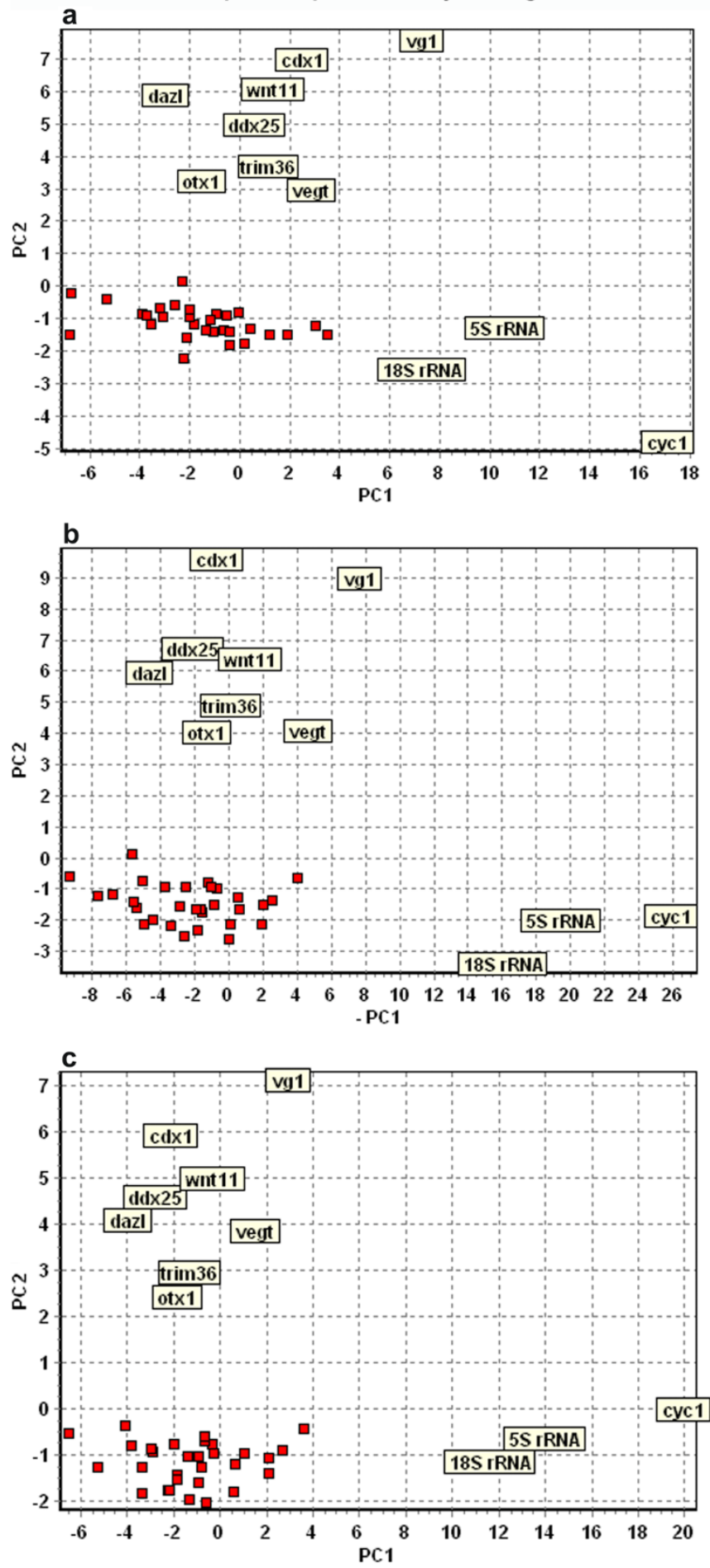

Figure 3 Principal component analysis of maternal genes. (A) 8-cell stage (four embryos/32 cells analyzed), (B) 16-cell stage (four embryos/64 cells analyzed), and (C) 32-cell stage (32 cells analyzed) embryos. Three clusters are seen in all the developmental stages. First cluster comprises $18 \mathrm{~S}$ rRNA, $5 S$ rRNA, and cycl (mitochondrial cytochrome c); second cluster vgl, $c d x 1$ (xcad2), wnt11, dazl, vegt, ddx25 (deadsouth), otx1, and trim36; third cluster (red squares) fzd7, par1, bmp2, pias1, dvl2, dvl3, lrp6, foxr1, fart1, mapk8, odc1, axin1, est1, U3 snoRNA, apc, gapdh, acta, eeflal, tcf3, zpc, RNA polymerase II, gsk3b, maml1, tubb, ctnnb1 ( $\beta$-catenin), mos, oct60, foxh1, stat 3 , and pax6. The animal-vegetal distinction is found along the $y-$ axis (PC2), while differences in expression level of the maternal transcripts is reflected along the $\mathrm{x}$-axis (PC1).
Animal Welfare under the Ministry of Agriculture of Czech Republic (accreditation No.24773/2008-10001, date of expiry 10.12.2013). The experiment was also approved by the institutional ethics committee.

In vitro fertilization and collection of single blastomeres. Two Xenopus laevis females were injected with $450 \mathrm{U}$ of human chorionic gonadotrophin hormone (hCG) and kept overnight at room temperature. Ovulated oocytes were obtained 12 hours after stimulation by manual squeezing. Testes of Xenopus laevis males were removed and homogenized in L-15 Leibowitźs medium with $15 \%$ of fetal bovine serum. Oocytes were in vitro fertilized by sperm suspension and $1 \times$ MMR medium (Marćs Modified Ringers medium: $0.1 \mathrm{M} \mathrm{NaCl}, 2.0 \mathrm{mM} \mathrm{KCl}, 1 \mathrm{mM} \mathrm{MgCl}, 2 \mathrm{mM}$ $\mathrm{CaCl}_{2}, 5$ mM HEPES, $\mathrm{pH}$ 7.7) was added to cover the fertilized oocytes. Jelly coats were removed by cysteine treatment ( $2.2 \%$ in MMR, for 8 minutes) at 20 minutes after fertilization. During cell division the embryos were transferred to $\mathrm{Ca}^{2+}$ and $\mathrm{Mg}^{2+}$ free medium ( $88 \mathrm{mM} \mathrm{NaCl}, 1 \mathrm{mM} \mathrm{KCl}, 2.4 \mathrm{mM} \mathrm{NaHCO}_{3}, 7.5 \mathrm{mM}$ Tris, $\left.\mathrm{pH} 7.7\right)$ and kept there for 60 minutes in order to separate single blastomeres. Vitelline membranes of the embryos containing 8,16 , and 32 cells were manually torn and individual blastomeres were collected. The blastomeres were collected separately from animal and vegetal hemispheres, but in random order to avoid introducing any technical bias and subjective dorso-ventral specification before measurement.

Isolation of total RNA and reverse transcription. The individual blastomeres collected from the 8 and 16-cell stages were homogenized in $200 \mu \mathrm{l}$ and blastomeres from the 32-cell stage in $100 \mu \mathrm{l}$ of TRIzol reagent (Invitrogen), and immediately frozen at $-80^{\circ} \mathrm{C}$. After thawing, the samples were carefully vortexed for 2 minutes to pulp yolk. Manufacturer's instructions were followed during isolation of total RNA, and glycogen was added to enhance RNA precipitation yield. RNA from individual cells from the 8 and 16-cell stages was dissolved in $20 \mu \mathrm{l}$ of RNase-DNase free water. Samples from the 32-cell stage were dissolved in $12 \mu \mathrm{l}$ of water. RNA concentration was measured using the Nanodrop ND1000 quantification system. cDNA was produced with SuperScript ${ }^{\mathrm{TM}}$ III Reverse transcriptase kit (Invitrogen). $10 \mathrm{ng}$ of total RNA, $0.5 \mu \mathrm{l}$ of a mixture of oligo-dT and random hexamers (mixture $1: 1,50 \mu \mathrm{M}$ each), $0.5 \mu \mathrm{l}$ of dNTPs (10 mM each), and $0.5 \mu \mathrm{l}$ of RNA spike (in vitro transcribed artificial RNA with $3^{\prime}$ polyA tail and 5' cap, TATAA Biocenter) were mixed with sterile water to a total volume of $6.5 \mu \mathrm{l}$. Samples were incubated for $5 \mathrm{~min}$ at $70^{\circ} \mathrm{C}$, followed by $20 \mathrm{sec}$ at $25^{\circ} \mathrm{C}$, and then cooled at $4^{\circ} \mathrm{C}$ for $1 \mathrm{~min} .100 \mathrm{U}$ of SuperScript III reverse transcriptase, $20 \mathrm{U}$ of RNaseOUT ${ }^{\mathrm{TM}}$ (recombinant ribonuclease inhibitor, Invitrogen), $0.5 \mu \mathrm{l}$ of $0.1 \mathrm{M} \mathrm{DTT}$ and $2 \mu \mathrm{l}$ of $5 \times$ first strand synthesis buffer was added to a final volume of $10 \mu \mathrm{l}$. The following temperature gradient profile was used to synthesize cDNA: $5 \mathrm{~min}$ at $25^{\circ} \mathrm{C}, 60 \mathrm{~min}$ at $50^{\circ} \mathrm{C}, 15 \mathrm{~min}$ at $55^{\circ} \mathrm{C}$, and $15 \mathrm{~min}$ at $75^{\circ} \mathrm{C}$. cDNA samples were diluted 8 times to a final volume of $80 \mu \mathrm{l}$ and were stored at $-20^{\circ} \mathrm{C}$.

Primer design and quantitative PCR. Primer assays for the selected 41 maternal genes (fzd7, otx1, bmp2, pias1, dvl2, dvl3, lrp6, trim36, foxr1, frat1, mapk8, odc1, $18 \mathrm{~S}$ rRNA, 5S rRNA, cyc1, acta, tubb, gapdh, maml1, eefla1, RNA polymerase II, U3 snoRNA, par1, oct60, ddx25, dazl, cdx1, wnt11, vg1, vegt, axin1, est1, apc, tcf3, zpc, gsk3b, ctnnb1, mos, foxh1, stat3, pax6) were designed with Primer3Plus (http:// www.bioinformatics.nl/cgi-bin/primer3plus/primer3plus.cgi). Annealing temperature was set to $60^{\circ} \mathrm{C}$ and the length of qPCR products was around $120 \mathrm{bp}$. Specificity of the assays was tested in silico using blast (http://blast.ncbi.nlm.nih.gov/ Blast.cgi). Primer sequences are available upon request. qPCR and melting curve validations were measured on the real-time CFX384 cycler system (BioRad). GPCR mix contained $2 \mu \mathrm{l}$ of cDNA, $0.5 \mu \mathrm{l}$ of forward and reverse primers (mixture $1: 1$, $10 \mu \mathrm{M}$ each), $5 \mu \mathrm{l}$ of SYBR Green JumpStart ${ }^{\mathrm{TM}}$ Taq Ready Mix ${ }^{\mathrm{TM}}$ (Sigma), and water in a total volume of $10 \mu \mathrm{l}$. The cycling program was: $2 \mathrm{~min}$ at $95^{\circ} \mathrm{C}$ for activation of the polymerase, followed by 40 cycles of denaturation at $95^{\circ} \mathrm{C}$ for $15 \mathrm{sec}$, annealing at $60^{\circ} \mathrm{C}$ for $20 \mathrm{sec}$ and elongation at $72^{\circ} \mathrm{C}$ for $30 \mathrm{sec}$. Post PCR melting curves were measured from 65 to $95^{\circ} \mathrm{C}$ in $0.5^{\circ} \mathrm{C}$ intervals to validate the formation of expected PCR products.

Data analysis. Data were analyzed with GenEx (MultiD, version 5.3.). Off scale data were removed during pre-processing using a cut off at 36 cycles and outliers were identified with Grubb's test. Missing data were imputed using the average Cq of the other blastomeres from the same hemisphere in the same embryo (roughly $6 \%$ of the data was imputed). All data were normalized to the spike and converted to relative quantities (relative to the highest $\mathrm{Cq}$ for each gene, corresponding to arbitrary assigning an expression of 1 to the least expressed sample). Last step of the preprocessing was to transform the data to $\log 2$ scale. 8-cell, 16-cell and 32-cell embryos were analyzed separately. The combined expression of the individual blastomeres adds up to the expression of the original oocyte, which is expected, since de novo expression does not start until MBT. Three multivariate statistical methods were used for classification: principal component analysis (PCA), Kohonen self-organizing map (SOM), and hierarchical clustering presented as heatmaps. PCA of individual blastomeres (Fig. 1) was performed on data autoscaled along genes, while heatmaps (Fig. 2) and PCA of genes (Fig. 3) were performed on data autoscaled along samples ${ }^{35}$.

1. Bengtsson, M., Ståhlberg, A., Rorsman, P. \& Kubista, M. Gene expression profiling in single cells from the pancreatic islets of Langerhans reveals lognormal distribution of mRNA levels. Genome Res. 15, 1388-1392 (2005). 
2. Guo, G. et al. Resolution of cell fate decisions revealed by single-cell gene expression analysis from zygote to blastocyst. Dev. Cell 18, 675-685 (2010).

3. May, A. et al. Multiplex RT-PCR expression analysis of developmentally important genes in individual mouse preimplantation embryos and blastomeres. Biol. Reprod. 80, 194-202 (2009).

4. Galan, A. et al. Functional genomics of 5- to 8-cell stage human embryos by blastomere single-cell cDNA analysis. PLoS One 5, e13615 (2010).

5. Virant-Klun, I. et al. Expression of pluripotency and oocyte-related genes in single putative stem cells from human adult ovarian surface epithelium cultured in vitro in the presence of follicular fluid. BioMed Res. Int. 2013, 1-18 (2012).

6. Danilchik, M. V. \& Gerhart, J. C. Differentiation of the animal-vegetal axis in Xenopus laevis oocytes. I. Polarized intracellular translocation of platelets establishes the yolk gradient. Dev. Biol. 122, 101-112 (1987).

7. King, M. L., Messitt, T. J. \& Mowry, K. L. Putting RNAs in the right place at the right time: RNA localization in the frog oocyte. Biol. Cell 97, 19-33 (2005).

8. Sindelka, R., Jonak, J., Hands, N., Bustin, S. A. \& Kubista, M. Intracellular expression profiles measured by real-time PCR tomography in the Xenopus laevis oocyte. Nucleic Acids Res. 36, 387-392 (2008).

9. Sindelka, R., Sidova, M., Svec, D. \& Kubista, M. Spatial expression profiles in the Xenopus laevis oocytes measured with qPCR tomography. Methods 51, 87-91 (2010).

10. Roth, S. The origin of dorsoventral polarity in Drosophila. Philos. Trans. R. Soc. Lond. B. Biol. Sci. 358, 1317-1329 (2003).

11. Aanstad, P. \& Whitaker, M. Predictability of dorso-ventral asymmetry in the cleavage stage zebrafish embryo: an analysis using lithium sensitivity as a dorsoventral marker. Mech. Dev. 88, 33-41 (1999).

12. Sive, L. H., Grainger, M. R. \& Harland, M. R. Early development of Xenopus laevis - A laboratory manual. Cold Spring Harbor Laboratory Press 2000, chapter 2, figure 2.1 .

13. Vincent, J. P. \& Gerhart, J. C. Subcortical rotation in Xenopus eggs: an early step in embryonic axis specification. Dev. Biol. 123, 526-539 (1987).

14. Denegre, J. M. \& Danilchik, M. V. Deep cytoplasmic rearrangements in axisrespecified Xenopus embryos. Dev. Biol. 160, 157-164 (1993).

15. Miller, J. R. et al. Establishment of the dorsal-ventral axis in Xenopus embryos coincides with dorsal enrichment of dishevelled that is dependent on cortical rotation. J. Cell Biol. 146, 427-437 (1999).

16. Marikawa, Y., Li, Y. \& Elinson, R. P. Dorsal determinants in the Xenopus egg are firmly associated with the vegetal cortex and behave like activators of the Wnt pathway. Dev. Biol. 191, 69-79 (1997).

17. Darras, S., Maikawa, Y., Elinson, R. P. \& Lemaire, P. Animal and vegetal pole cells of early Xenopus embryos respond differently to maternal dorsal determinants: implications for the patterning of the organiser. Development 124, 4275-4286 (1997).

18. Hyatt, B. A., Lohr, J. L. \& Yost, H. J. Initiation of vertebrate left-right axis formation by maternal Vg1. Nature 384, 62-65 (1996).

19. King, M. L., Messitt, T. J. \& Mowry, K. L. Putting RNAs in the right place at the right time: RNA localization in the frog oocyte. Biol. Cell 97, 19-33 (2005).

20. Kofron, M. et al. New roles for FoxH1 in patterning the early embryo. Development 131, 5065-5078 (2004)

21. Kofron, M. et al. Wnt11/ $\beta$-catenin signaling in both oocytes and early embryos acts through LRP6-mediated regulation of axin. Development 134, 503-513 (2007).

22. Cuykendall, T. N. \& Houston, D. W. Identification of germ plasm-associated transcripts by microarray analysis of Xenopus vegetal cortex. Dev. Dyn. 239, 1838-1848 (2010).

23. Cuykendall, T. N. \& Houston, D. W. Vegetally localized Xenopus trim 36 regulates cortical rotation and dorsal axis formation. Development 136, 3057-3065 (2009).
24. Bergkvist, A. et al. Gene expression profiling - Clusters of possibilities. Methods 50, 323-335 (2010).

25. Keller, R. E. Vital dye mapping of the gastrula and neurula of Xenopus laevis: I. Prospective areas and morphogenetic movements of the superficial layer. Dev. Biol. 42, 222-241 (1975).

26. Keller, R. E. Vital dye mapping of the gastrula and neurula of Xenopus laevis: II. Prospective areas and morphogenetic movements of the deep layer. Dev. Biol. 51, 118-137 (1976)

27. Kikkawa, M., Takano, K. \& Shinagawa, A. Location and behavior of dorsal determinants during first cell cycle in Xenopus eggs. Development 122, 3687-3696 (1996).

28. Weaver, C. \& Kimelman, D. Move it or lose it: axis specification in Xenopus. Development 131, 3491-3499 (2004).

29. Medina, A., Wendler, S. R. \& Steinbeisser, H. Cortical rotation is required for the correct spatial expression of nr3, sia and gsc in Xenopus embryos. Int. J. Dev. Biol. 41, 741-745 (1997).

30. Kugler, J. M. \& Lasko, P. Localization, anchoring and translational control of oskar, gurken, bicoid and nanos mRNA during Drosophila oogenesis. Fly (Austin) 3, 15-28 (2009)

31. Steinhauer, J. \& Kalderon, D. Microtubule polarity and axis formation in the Drosophila oocyte. Dev. Dyn. 235, 1455-1468 (2006).

32. Hainski, A. M. \& Moody, S. A. Xenopus maternal mRNAs from dorsal animal blastomere induce a secondary axis in host embryo. Development 116, 347-355 (1992).

33. Tao, Q. et al. Maternal Wnt11 activates the canonical Wnt signaling pathway required for axis formation in Xenopus embryos. Cell 120, 857-871 (2005).

34. Schroeder, K. E., Condic, M. L., Eisenberg, L. M. \& Yost, H. J. Spatially regulated translation in embryos: asymmetric expression of maternal wnt-11 along the dorso-ventral axis in Xenopus. Dev. Biol. 214, 288-297 (1999).

35. Ståhlberg, A., Rusnakova, V., Forootan, A., Anderova, M. \& Kubista, M. RT-qPCR work-flow for single-cell data analysis. Methods 59, 80-88 (2013).

\section{Acknowledgements}

This study was supported by Grant agency of Czech Republic 301/09/1752; Ministry of Youth, Education and Sports of the Czech Republic AV0Z50520701; BIOCEV CZ.1.05/ 1.1.00/02.0109 from the ERDF and by the grant SVV-2013-267212.

\section{Author contributions}

M.F. performed the experiments and together with M.K. analyzed the obtained data. R.S. and M.K. conceived and designed the experiments. All authors co-wrote the manuscript and approved the final paper.

\section{Additional information}

Supplementary information accompanies this paper at http://www.nature.com/ scientificreports

Competing financial interests: The authors declare no competing financial interests.

How to cite this article: Flachsova, M., Sindelka, R. \& Kubista, M. Single blastomere expression profiling of Xenopus laevis embryos of 8 to 32-cells reveals developmental asymmetry. Sci. Rep. 3, 2278; DOI:10.1038/srep02278 (2013).

This work is licensed under a Creative Commons AttributionNonCommercial-NoDerivs 3.0 Unported license. To view a copy of this license, visit http://creativecommons.org/licenses/by-nc-nd/3.0 